\title{
Impaired cough suppression in chronic refractory cough
}

\author{
Peter S.P. Cho (1) ${ }^{1}$, Hannah V. Fletcher ${ }^{2}$, Richard D. Turner (10 ${ }^{1,3}$, \\ Caroline J. Jolley ${ }^{1}$ and Surinder S. Birring ${ }^{1,2}$
}

Affiliations: ${ }^{1}$ Centre for Human and Applied Physiological Sciences, School of Basic and Medical Biosciences, King's College London, London, UK. 'Dept of Respiratory Medicine, King's College Hospital NHS Foundation Trust, London, UK. ${ }^{3}$ Dept of Respiratory Medicine, Charing Cross Hospital, Imperial College Healthcare Trust, London, UK.

Correspondence: Surinder S. Birring, Dept of Respiratory Medicine, Chest Unit, Cheyne Wing, King's College Hospital, Denmark Hill, London, SE5 9RS, UK. E-mail: surinder.birringanhs.net

\section{@ERSpublications}

Individuals with chronic refractory cough (CRC) are less able to suppress cough during a capsaicin cough suppression test (CST). The CST is repeatable, associated with objective cough frequency and can distinguish patients with CRC from healthy subjects. http://ow.ly/S8Ki30nSaio

Cite this article as: Cho PSP, Fletcher HV, Turner RD, et al. Impaired cough suppression in chronic refractory cough. Eur Respir J 2019; 53: 1802203 [https://doi.org/10.1183/13993003.02203-2018].

ABSTRACT Functional brain imaging in individuals with chronic cough demonstrates reduced activation in cortical regions associated with voluntary cough suppression. Little is known about the ability of patients with chronic cough to suppress cough. This study aimed to compare the ability to voluntarily suppress cough during inhaled capsaicin challenge in participants with chronic refractory cough with that in healthy controls. In addition, this study aimed to assess the repeatability of capsaicin challenge test with voluntary cough suppression.

Participants with chronic refractory cough and healthy controls underwent inhaled capsaicin challenge tests while attempting to suppress their cough responses. After 5 days, either a conventional capsaicin challenge test with no cough suppression attempt, or a repeat test with an attempt at cough suppression was performed. Threshold capsaicin concentrations required to elicit one, two and five coughs were calculated by interpolation. Objective 24 -h cough frequency was measured in individuals with chronic refractory cough.

Healthy controls were able to suppress capsaicin-evoked cough while participants with chronic refractory cough were not. Geometric mean \pm SD capsaicin dose thresholds for five coughs with (CS5) and without (C5) suppression attempts were $254.40 \pm 3.78$ versus $45.89 \pm 3.95 \mu \mathrm{mol} \cdot \mathrm{L}^{-1}$, respectively, in healthy controls $(\mathrm{p}=0.033)$ and $3.34 \pm 5.04$ versus $3.86 \pm 5.13 \mu \mathrm{mol} \cdot \mathrm{L}^{-1}$, respectively, in participants with chronic refractory cough $(\mathrm{p}=0.922)$. Capsaicin dose thresholds for triggering five coughs with self-attempted cough suppression were significantly lower in participants with chronic refractory cough than in healthy controls; geometric mean \pm SD $4.94 \pm 4.43$ versus $261.10 \pm 4.34 \mu \mathrm{mol} \cdot \mathrm{L}^{-1}$, respectively; mean difference (95\% CI) 5.72 (4.54-6.91) doubling doses $(\mathrm{p}<0.001)$. Repeatability of cough suppression test in both patients and healthy controls was high; intraclass correlation coefficients of $\log (\mathrm{CS} 5)$ values 0.81 and 0.87 , respectively. CS5 was associated with objective cough frequency $(\rho=-0.514, p=0.029)$.

Participants with chronic refractory cough were less able to voluntarily suppress capsaicin-evoked cough compared to healthy controls. This may have important implications for the pathophysiology and treatment of chronic cough. 


\section{Introduction}

Chronic cough, defined as a cough lasting $>8$ weeks in duration, affects up to $9.6 \%$ of the population $[1,2]$. In up to $42 \%$ of patients, the cough remains persistent despite extensive investigations and trials of treatment; this is often referred to as chronic refractory cough (CRC) [3, 4]. CRC is associated with considerable physical and psychological morbidity [5-7]. The mechanism of cough in CRC is unclear. The symptom profile of patients and the observation of hypersensitivity to tussive agents, such as capsaicin, had led to the hypothesis that CRC is a disorder of dysfunctional airway sensory nerves and their central processing [8, 9]. In 2009, ECCLES [10], and more recently HeGLAND et al. [11] proposed that the voluntary suppression of cough is an important mechanism in the regulation of cough. ANDO et al. [12] have demonstrated using functional neuroimaging in chronic cough that there is reduced activity in forebrain regions including the dorsomedial, prefrontal and anterior mid-cingulate cortices. These same areas appear engaged in the voluntary suppression of capsaicin-evoked cough [12-14].

Cough suppression can be studied by modifying the capsaicin challenge test to ask participants to attempt to prevent themselves from coughing following inhalation of the tussive agent $[15,16]$. Although this technique has demonstrated that healthy subjects can suppress capsaicin-evoked cough [15], little is known about whether those with CRC can do the same. We hypothesised that patients with CRC are less able to voluntarily suppress cough during a capsaicin challenge test compared to healthy subjects.

We investigated the feasibility and repeatability of a cough suppression test in participants with CRC and healthy subjects. We investigated and compared the ability of self-attempted cough suppression during capsaicin challenge test between participants with CRC and healthy controls. We also investigated the relationship of the ability to suppress cough with 24 -h objective cough frequency and health status. Lastly, we investigated the ability of the cough suppression test to differentiate patients with CRC from healthy controls.

\section{Methods}

Participants

Patients with chronic refractory cough ( $>8$ weeks' duration) were recruited prospectively from a tertiary care specialist cough clinic (King's College Hospital, London, UK). The diagnosis of chronic refractory cough was assessed by clinicians according to the British Thoracic Society guidelines for the management of chronic cough in adults [17]. Inclusion criteria were a diagnosis of chronic cough, either unexplained or refractory to treatment of a known potential cause and a normal chest radiograph. Exclusion criteria were the presence of another chronic respiratory disease, use of angiotensin-converting enzyme inhibitor medication within the past 12 months, current smoking, smoking within the past 12 months and upper respiratory tract infection within the past 4 weeks.

Healthy controls were recruited prospectively through local advertisement. Exclusion criteria were identical to those for participants with chronic refractory cough with the addition of the presence of cough in the past 8 weeks, and a ratio of forced expiratory volume in $1 \mathrm{~s}$ to forced vital capacity $<0.7$. All participants gave informed written consent. The study was granted research ethics committee approval (National Research Ethics Service East London and The City, 10/H0703/6). All participants provided written informed consent for participation in the study. All ethical elements of the study conformed to the Declaration of Helsinki.

\section{Protocol}

Participants had no prior exposure to a capsaicin challenge test. Participants underwent investigations over two visits. At visit 1, demographic and anthropometric data were collected. All participants underwent spirometry and a modified capsaicin challenge test with attempts at voluntary cough suppression, "cough suppression test". In addition, participants with CRC completed subjective assessments of cough severity, urge to cough, cough-specific quality of life, anxiety and depression. Participants with CRC were invited to undergo 24-h objective cough monitoring. At visit 2, which followed visit 1 by 5 days, participants were selected at random to undergo either a standard capsaicin challenge test without attempting to suppress coughing or a repeat cough suppression test (supplementary figure E1). An interval of 5 days was chosen between visits 1 and 2 to avoid the potential for tachyphylaxis to capsaicin [18].

\section{Capsaicin challenge test}

Cough reflex sensitivity was assessed as per the recommendations of the European Respiratory Society (ERS) guidelines [19]. Capsaicin (Sigma-Aldrich, St. Louis, MO, USA) solution was administered as $10-\mu \mathrm{L}$ single breath inhalations via an air-powered dosimeter (KoKo Digidoser; nSpire Health, Longmont, CO, USA) at increasing doubling doses $\left(0.49-1000 \mu \mathrm{mol} \cdot \mathrm{L}^{-1}\right)$ at $1-\mathrm{min}$ intervals. $0.9 \%$ saline solutions were interspersed randomly to reduce the effect of anticipation [19, 20]. A single characterised nebuliser 
(DeVilbiss Healthcare, New York, NY, USA) with an output of $1.205 \mathrm{~mL} \cdot \mathrm{min}^{-1}$ was utilised for all participants throughout the study. A valve was utilised to restrict the inspiratory flow to $0.5 \mathrm{~L} \cdot \mathrm{s}^{-1}[19,21]$. A minimum of three respiratory cycles were performed prior to the administration of each solution. The inspiratory-expiratory flow-volume signals were inspected in real time by two operators (P.S.P. Cho and H.V. Fletcher) to ensure a consistent inspiratory effort throughout the administration of the nebulised solution. If the participant did not take a full inhalation as observed during the real-time visual display of the flow-volume signal, the test was repeated. The number of coughs elicited by each inhalation was counted with the aid of a digital audio recorder (ICD-PX333; Sony Corporation, Tokyo, Japan) for $15 \mathrm{~s}$ after each dose administration [19, 21]. The challenge test continued until five or more coughs were elicited by a single inhalation of solution.

Modified capsaicin tussive challenge ("cough suppression test")

The ability to suppress cough was assessed by modifying the capsaicin challenge test; participants were instructed, "Please do not cough during the test". The capsaicin concentrations required to elicit one cough (CS1), two coughs (CS2) and five coughs (CS5) were calculated by interpolation [8].

\section{Standard capsaicin tussive challenge (without self-attempted cough suppression)}

During a standard capsaicin challenge test, participants were instructed, "Please cough if you wish during the test". The capsaicin concentrations required to elicit one cough $(\mathrm{C} 1)$, two coughs $(\mathrm{C} 2)$ and five coughs (C5) were calculated by interpolation [8].

\section{Cough frequency monitoring}

Cough frequency was recorded for $24 \mathrm{~h}$ using the Leicester Cough Monitor (LCM) [22]. The LCM is a validated ambulatory cough monitoring system which consists of an MP3 audio recorder (ICD-PX333), free-field microphone (LFH9173; Philips, Amsterdam, the Netherlands) and cough detection software [22]. Coughs were detected as single events regardless of whether they occurred in isolation or in bouts [22]. Both awake cough counts (number of coughs per estimated time spent awake) and awake daily cough frequency (coughs per hour) were documented. The participants were requested to record and report their time spent asleep.

\section{Subjective assessments}

Cough severity, urge to cough and health status

Cough severity and urge to cough were recorded on visual analogue scales (VAS) (range 0-100 mm; higher scores indicate more severe cough and more severe urge) [19]. The health status of the participants with CRC was recorded using the Leicester Cough Questionnaire (LCQ), which is a validated self-administered questionnaire for cough-specific health status in chronic cough (range 3-21; higher scores indicate better health status) [23].

\section{Depression and anxiety}

The Patient Health Questionnaire (PHQ9) was used to assess the severity of depression (range 0-27; higher scores indicate more severe depression) [24]. The Generalised Anxiety Disorder Assessment (GAD7) (range 0-21; higher scores indicate more severe anxiety), a validated self-administered questionnaire, was utilised to assess the severity of generalised anxiety [25].

\section{Lung function}

Spirometry (Jaeger MS-PFT Analyser Unit with Sentry Suite software version 2.19.96; Vyaire Medical, Mettawa, IL, USA) was measured according to the guidelines of the ERS and the American Thoracic Society [26].

\section{Statistical analysis}

The distribution of data was assessed using the D'Agostino-Pearson test. Parametric data were expressed as mean $\pm \mathrm{SD}$, whereas nonparametric data were expressed as median (interquartile range (IQR)). The capsaicin challenge and cough frequency data were presented as geometric mean \pm SD. Parametrically distributed data were analysed with paired t-test to compare sample means for paired data, and the independent unpaired Welch's t-tests to compare sample means for unpaired data. Comparison of nonparametric data was carried out using the Wilcoxon matched-pairs signed rank test for paired data, and the Mann-Whitney U-test for unpaired data. Fisher's exact test and the Chi-squared test were utilised for categorical data. Correlations between variables were analysed with Spearman's correlation coefficient ( $\rho$ ) for nonparametric data. Repeatability was assessed using the Bland-Altman method and intraclass correlation coefficients (ICC) based on a single-rater, absolute agreement, two-way mixed-effects model. 
The concentrations of capsaicin required to elicit one, two and five coughs were calculated by interpolation of the $\log$ dose-response curve [8]. A value of $1000 \mu \mathrm{mol} \cdot \mathrm{L}^{-1}$ was assigned to any interpolated values which were $>1000 \mu \mathrm{mol} \cdot \mathrm{L}^{-1}$. Standard capsaicin provocation test end-points were expressed as C1, C2 and C5, which were the capsaicin concentrations required to elicit 1,2 and 5 coughs respectively. Cough suppression test end-points were expressed as CS1, CS2 and CS5; the capsaicin concentrations required to elicit one, two and five coughs, respectively, while participants attempted to self-suppress coughing.

The sensitivity and specificity of the cough suppression test and standard capsaicin challenge test for distinguishing patients with cough from healthy controls were analysed using receiver operator characteristic (ROC) curve analysis. Youden's index [27] was used to identify the optimal threshold. p-values $<0.05$ were considered statistically significant.

From a previous study, we expected $\geqslant 10$ participants to be a sufficient sample size for making intra-individual comparisons in a tussive challenge test [21]. Therefore, a minimum of 10 patients and controls were needed for each test option in visit 2. Therefore, we aimed to recruit a sample size of 20-30 participants with CRC and healthy controls.

All analyses were performed on Prism version 7.0c (GraphPad Software, San Diego, CA, USA), except the Bland-Altman, ICC correlations and ROC curve analyses, which were performed on RStudio version 1.1.383 (RStudio Inc., Boston, MA, USA) for macOS version 10.14.

\section{Results}

\section{Participant characteristics}

30 consecutive participants with CRC were recruited and compared with 23 healthy controls; demographics, anthropometrics, spirometry and clinical characteristics are shown in table 1 . There was no significant difference in age and sex between the participants with CRC and the healthy controls (table 1). The median (IQR) duration of cough in participants with CRC was 7.0 (2.3-20.0) years. No participants in the study had a clinical diagnosis of depression or anxiety. A subgroup of 11 participants with CRC and

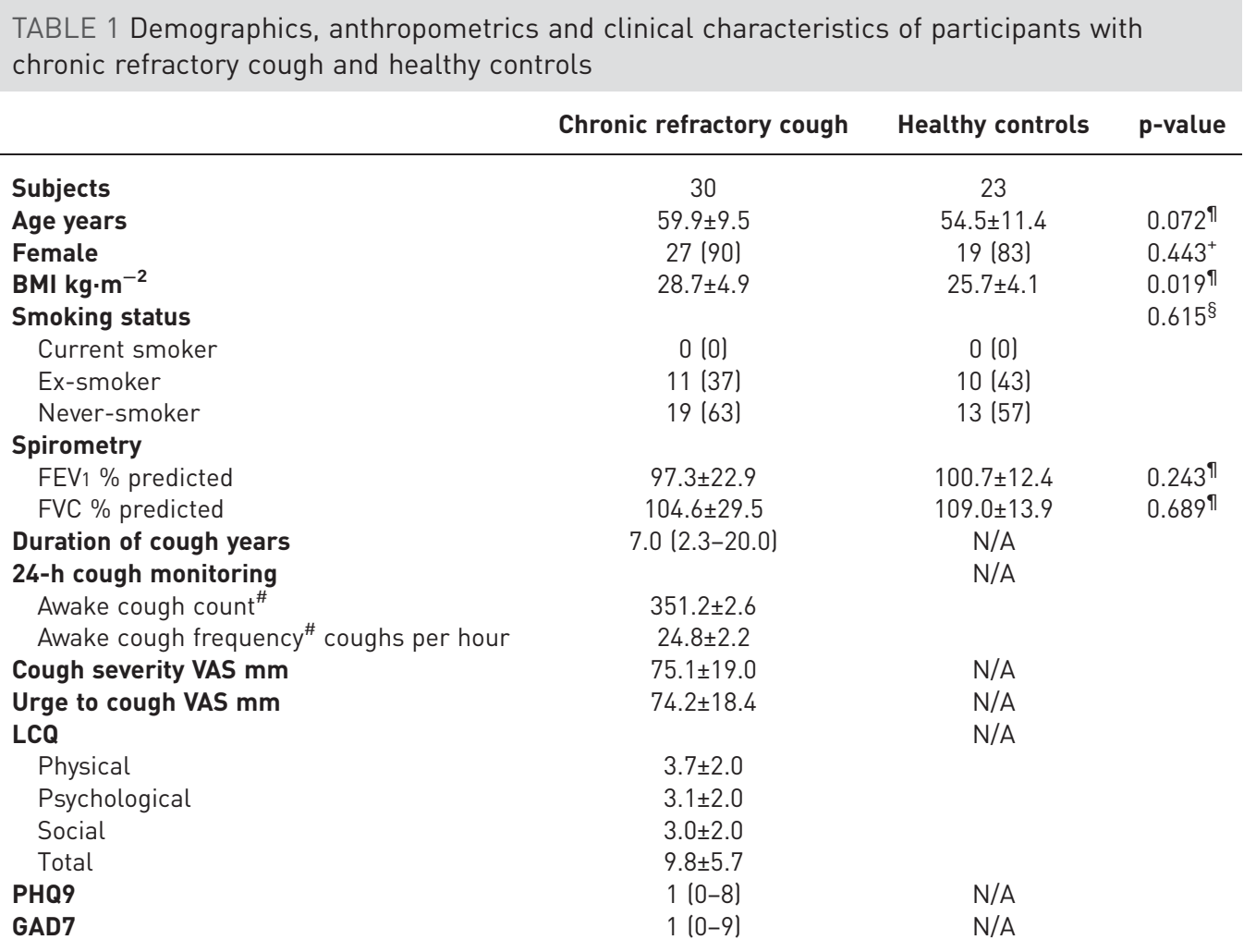

Data presented as $\mathrm{n}$, mean $\pm \mathrm{SD}, \mathrm{n}(\%)$ or median (interquartile range), unless otherwise stated. BMI: body mass index; FEV1: forced expiratory volume in $1 \mathrm{~s}$; FVC: forced vital capacity; VAS: visual analogue scale; LCQ: Leicester Cough Questionnaire; PHQ9: Patient Health Questionnaire; GAD7: Generalised Anxiety Disorder Assessment; N/A: not applicable. ${ }^{\#}$ : geometric mean $\pm S D$; ๆ: Welch's t-test; ${ }^{+}$: Fisher's exact test; §: Chi-squared test. 
13 healthy controls underwent the standard capsaicin provocation test, permitting coughing ad libitum, at a second visit. A subgroup of 13 participants with CRC and 10 healthy controls returned for a second visit to investigate the repeatability of the cough suppression test.

\section{Cough suppression test}

When attempting to suppress cough, capsaicin cough thresholds (CS1, CS2 and CS5) were significantly lower in participants with CRC compared to healthy controls (table 2 and figure 1). The mean difference (95\% CI) in CS5 between participants with CRC and healthy controls was 5.72 (4.54-6.91) doubling doses $(\mathrm{p}<0.001)$ (table 2 and figure 1$).$

\section{Standard capsaicin cough challenge}

When not attempting to suppress cough, participants with CRC again had significantly lower cough thresholds (C1, C2 and C5) than healthy controls (table 2). The mean difference (95\% CI) in C5 between participants with CRC and healthy controls was lower than the difference in CS5 between groups at 3.66 $(1.80-5.52)$ doubling doses $(\mathrm{p}<0.001)$ (table 2$)$.

\section{A comparison of cough suppression and standard capsaicin challenge test}

Healthy participants were able to suppress capsaicin-induced cough; a significant increase in the concentration of capsaicin required to elicit one, two and five coughs when asking subjects to attempt cough suppression compared to allowing coughing as desired: geometric mean \pm SD CS5 versus C5 254.40 \pm 3.78 versus $45.89 \pm 3.95 \mu \mathrm{mol} \cdot \mathrm{L}^{-1}$; mean difference (95\% CI) 2.77 (1.25-4.28) doubling doses ( $\left.\mathrm{p}=0.033\right)$ (figure 2a). In contrast, participants with CRC were unable to suppress capsaicin-induced cough, as similar capsaicin thresholds in both tests were demonstrated: geometric mean \pm SD CS5 versus C5 $3.34 \pm 5.04$ versus $3.86 \pm 5.13 \mu \mathrm{mol} \cdot \mathrm{L}^{-1}$; mean difference $(95 \% \mathrm{CI})-0.21(-1.37-0.96)$ doubling doses $(\mathrm{p}=0.922)$ (figure $\left.2 \mathrm{~b}\right)$.

\section{Repeatability of cough suppression}

In healthy controls, the ICCs for repeatability of $\log (\mathrm{CS} 1), \log (\mathrm{CS} 2)$ and $\log (\mathrm{CS} 5)$ were $0.64,0.83$ and 0.87 , respectively (table 3$)$. The mean difference $(95 \%$ CI) in CS5 in healthy controls was $0.04(-0.47-0.54)$ doubling doses over 5 days (figure 3a). The ICCs for repeatability of $\log (\mathrm{CS} 1), \log (\mathrm{CS} 2)$ and $\log (\mathrm{CS} 5)$ in participants with chronic refractory cough were $0.13,0.24$ and 0.81 , respectively (table 3 ). The mean difference $(95 \% \mathrm{CI})$ in CS5 in participants with CRC was $0.42(-1.37-2.22)$ doubling doses over 5 days (figure 3b). CS5 was considered the most repeatable end-point in both healthy controls and participants with CRC. There was no evidence that the outcome of the first cough suppression test influenced the second.

\section{Relationship between cough suppression ability and daily cough frequency}

18 participants with $\mathrm{CRC}$ agreed to undergo 24-h cough monitoring. The geometric mean \pm SD awake cough count was $351.2 \pm 2.6$ coughs over a 24 -h period (table 1 ). There were significant correlations between awake cough counts and CS1, CS2 and CS5 ( $\rho=-0.556, p=0.017 ; \rho=-0.551, p=0.018$; and $\rho=-0.514, p=0.029$, respectively) (figure 4 ).

\begin{tabular}{|c|c|c|c|}
\hline & Chronic refractory cough & Healthy controls & p-value ${ }^{\#}$ \\
\hline \multicolumn{4}{|c|}{ With self-attempted cough suppression } \\
\hline Subjects & 30 & 23 & \\
\hline $\mathrm{CS} 1 \mu \mathrm{mol} \cdot \mathrm{L}^{-1}$ & $2.01 \pm 3.40$ & $40.34 \pm 6.64$ & $<0.0001$ \\
\hline $\mathrm{CS} 2 \mu \mathrm{mol} \cdot \mathrm{L}^{-1}$ & $2.52 \pm 3.44$ & $71.52 \pm 6.06$ & $<0.0001$ \\
\hline $\mathrm{CS} 5 \mu \mathrm{mol} \cdot \mathrm{L}^{-1}$ & $4.94 \pm 4.43$ & $261.10 \pm 4.34$ & $<0.0001$ \\
\hline \multicolumn{4}{|c|}{ Without self-attempted cough suppression } \\
\hline Subjects & 11 & 13 & \\
\hline C1 $\mu \mathrm{mol} \cdot \mathrm{L}^{-1}$ & $0.91 \pm 5.17$ & $7.20 \pm 2.82$ & 0.0012 \\
\hline $\mathrm{C} 2 \mu \mathrm{mol} \cdot \mathrm{L}^{-1}$ & $1.31 \pm 4.89$ & $11.44 \pm 2.82$ & 0.0015 \\
\hline C5 $\mu \mathrm{mol} \cdot \mathrm{L}^{-1}$ & $3.86 \pm 5.13$ & $45.89 \pm 3.95$ & 0.0004 \\
\hline
\end{tabular}

Data presented as $\mathrm{n}$ or geometric mean \pm SD, unless otherwise stated. CS1, CS2, CS5: capsaicin concentrations to elicit one, two and five coughs, respectively, during self-attempted suppression of coughing; C1, C2, C5: capsaicin concentrations to elicit one, two and five coughs, respectively, without self-attempted cough suppression. " : Mann-Whitney U-test. 
FIGURE 1 Threshold capsaicin concentrations required to elicit five coughs in tussive challenges with self-attempted cough suppression in participants with chronic refractory cough and healthy controls.

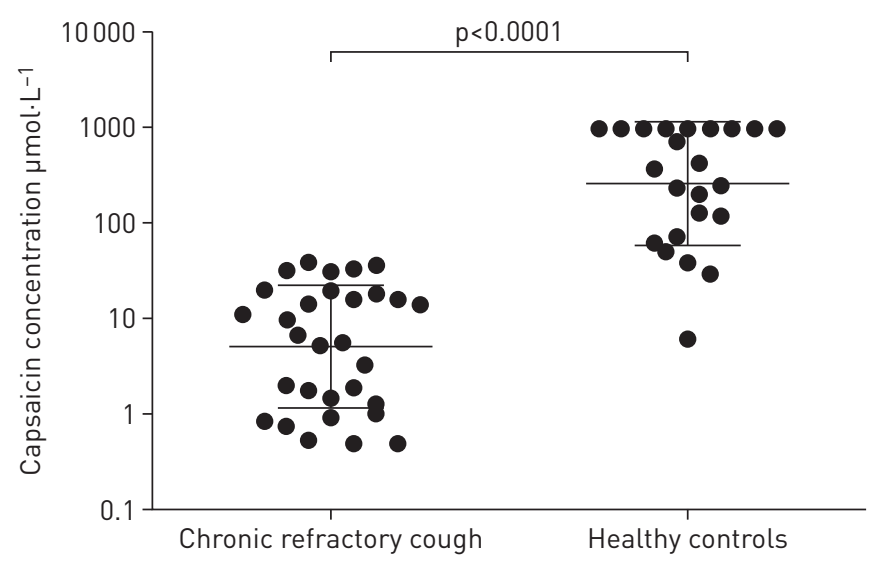

\section{Subjective assessments}

Cough severity, urge to cough and health status

Participants with CRC had mean \pm SD cough severity VAS scores $74.8 \pm 18.7 \mathrm{~mm}$, mean $\pm \mathrm{SD}$ urge to cough VAS scores $74.2 \pm 18.4 \mathrm{~mm}$ and mean \pm sD LCQ total scores $10.1 \pm 5.7$. There was no association between voluntary cough suppression test thresholds (CS1, CS2 and CS5) and either cough severity, urge to cough or health status scores (supplementary table E1).

\section{Depression and anxiety}

The median (IQR) PHQ9 and GAD7 scores for participants with CRC were $1(0-8)$ and 1 (0-9), respectively (table 1). $60 \%$ of participants reported no depressive symptoms on the PHQ9, while $26 \%$, $7 \%$ and $7 \%$ of participants reported mild, moderate or severe depressive symptoms, respectively. $74 \%$ of participants with CRC reported no anxiety on the GAD7 assessment, while $13 \%$ and 13\% participants reported mild or severe anxiety symptoms, respectively. There was no correlation between cough suppression thresholds (CS1, CS2 and CS5) and either PHQ9 or GAD7 scores ( $\rho=0.117-0.438$, $\mathrm{p}=0.104-0.676)$.

Optimal capsaicin cough thresholds to distinguish participants with CRC from healthy controls

CS5 was the most repeatable cough suppression end-point and was therefore selected for further analyses (figure 2 and table 3). The optimal thresholds for CS5 and C5 according to Youden's index were $\leqslant 38.86 \mu \mathrm{mol} \cdot \mathrm{L}^{-1}$ (sensitivity $=100.0 \%$ and specificity=91.3\%) and $\leqslant 12.59 \mu \mathrm{mol} \cdot \mathrm{L}^{-1}$ (sensitivity $=81.8 \%$ and specificity $=91.7 \%$ ), respectively (figure $5 \mathrm{a}$ and $\mathrm{b}$, supplementary table E2).

\section{Discussion}

We investigated the ability of patients with CRC to suppress their cough during a capsaicin challenge test. Patients with CRC were unable to suppress capsaicin-invoked cough compared to healthy subjects. The
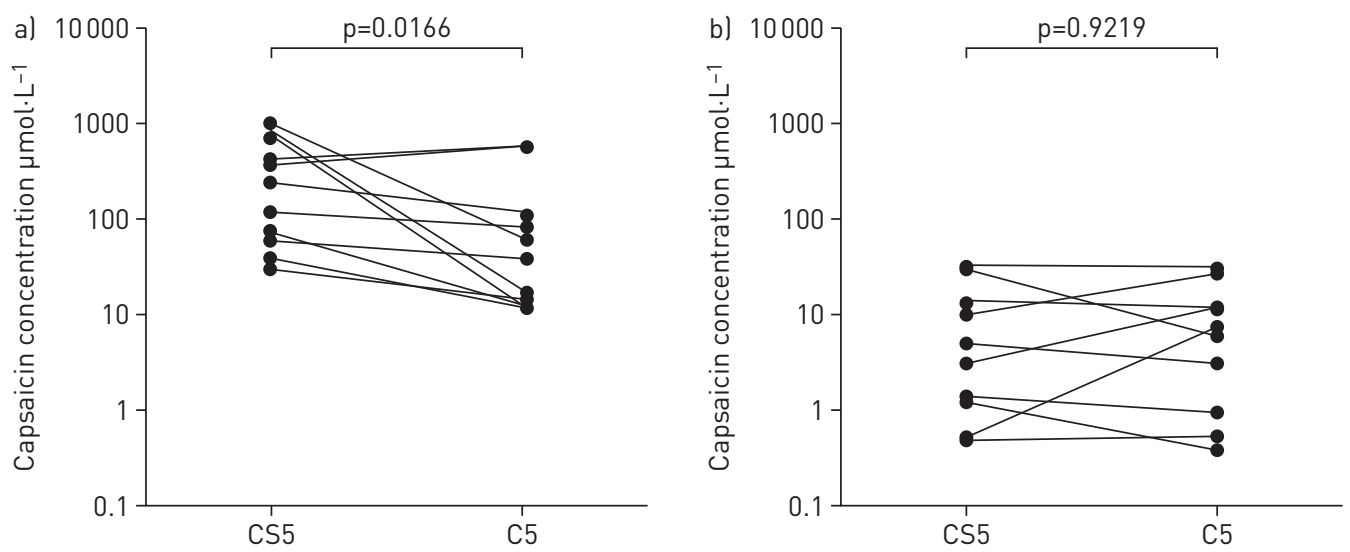

FIGURE 2 Threshold capsaicin concentrations required to elicit five coughs in tussive challenges with (CS5) and without (C5) self-attempted cough suppression in participants with chronic refractory cough and healthy controls. a) Healthy controls; b) chronic refractory cough. 
TABLE 3 Repeatability of self-attempted cough suppression in participants with chronic refractory cough and healthy controls

$$
\text { Day } 1
$$

Day 5

ICC (repeatability)

$2.00 \pm 4.69$
$2.71 \pm 4.00$
$6.79 \pm 4.17$

$40.17 \pm 4.13$
$96.08 \pm 1.18$
$361.60 \pm 2.87$

$2.00 \pm 4.69$

CS1 $\mu \mathrm{mol} \cdot \mathrm{L}^{-1}$

CS2 $\mu \mathrm{mol} \cdot \mathrm{L}^{-}$

CS5 $\mu \mathrm{mol} \cdot \mathrm{L}^{-1}$

Healthy controls

CS1 $\mu \mathrm{mol} \cdot \mathrm{L}^{-1}$

CS2 $\mu \mathrm{mol} \cdot \mathrm{L}^{-1}$

CS5 $\mu \mathrm{mol} \cdot \mathrm{L}^{-1}$
$1.27 \pm 17.44$

$2.08 \pm 9.38$

$9.11 \pm 4.02$

$69.53 \pm 4.18$

$89.83 \pm 4.37$

$370.90 \pm 3.09$
0.13

0.24

0.81

0.56

0.76

0.87

Data presented as geometric mean \pm SD, unless otherwise stated. ICC: intraclass correlation coefficient; CS1, CS2, CS5: capsaicin concentrations to elicit one, two or five coughs, respectively, with self-attempted cough suppression.

CS5 measure of cough suppression was highly repeatable and significantly more repeatable than CS1 and CS2. CS5 was associated with 24-h objective cough frequency in patients with chronic refractory cough. CS5 was better than C5 for distinguishing patients with CRC from healthy controls; a threshold of $39 \mu \mathrm{mol} \cdot \mathrm{L}^{-1}$ had a high sensitivity and specificity.

The key finding from our study was that patients with CRC were less able to suppress capsaicin-evoked cough compared to healthy subjects. In a small study investigating the effects of mindfulness meditation on cough, Young et al. [28] also demonstrated that patients with CRC could not suppress their cough as effectively as healthy subjects. An impairment in the ability to suppress cough suggests an abnormality in central neural pathways in patients with CRC. A recent study by ANDO et al. [12], utilising functional magnetic resonance imaging found reduced activity in the dorsomedial prefrontal cortex and anterior mid-cingulate cortices in the brain in patients with CRC when they were asked to suppress the urge to cough. ANDO et al. [12] proposed that a reduced capacity to suppress cough motor behaviour was an important component of the central neurobiology of cough hypersensitivity as well as the amplification of cough sensory inputs. A better understanding of the central neural mechanisms of cough may yield novel targets for developing antitussive therapy. It is likely that some patients will benefit from antitussive therapy that target central rather than peripheral mechanisms. Inhibitors of the sensory nerve P2X3 ion channel (MK7264) are the most promising peripherally acting antitussive therapy in development, but they are not effective in $30-40 \%$ of subjects, and the frequency of cough in those who benefit often does not return to normal levels [29]. There is a wide range of peripheral cough receptors and sensory nerves that mediate cough, and therefore it is likely that targeting one pathway will not benefit all patients [30]. Antitussive therapies that act centrally to inhibit the amplification of cough or enhance the activity of inhibitory neurons and their mediators have the advantage of intervening at a level where multiple peripheral inputs converge. Speech therapy and physiotherapy interventions that train patients to suppress
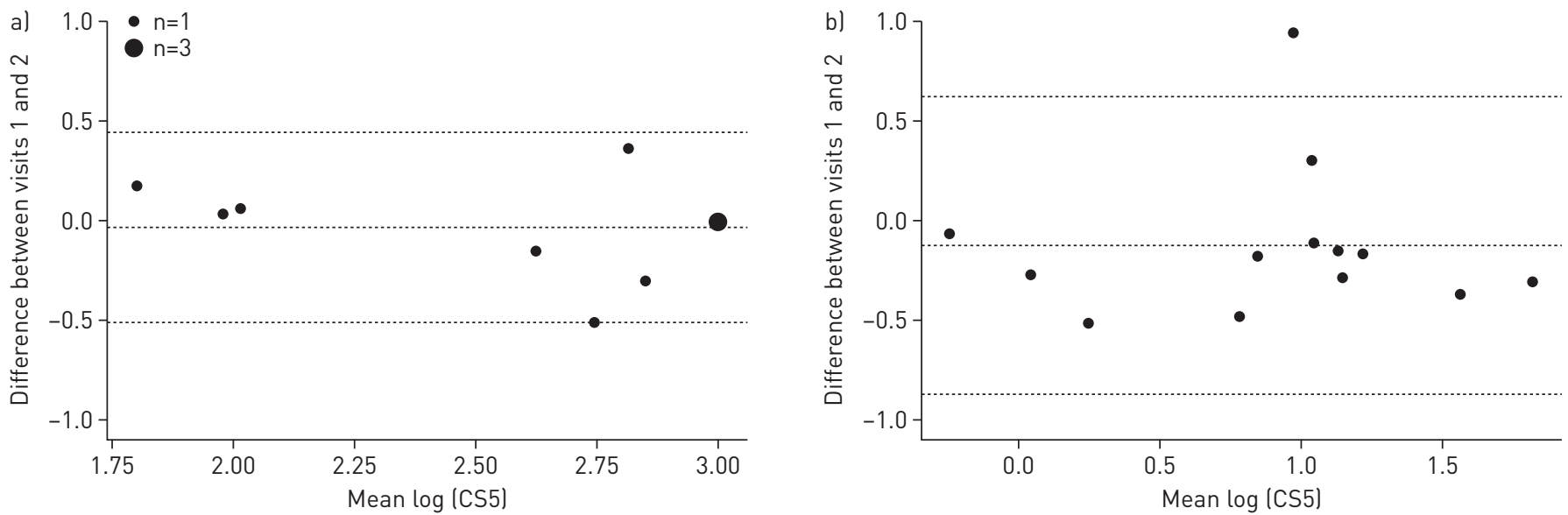

FIGURE 3 Bland-Altman plots evaluating repeatability of measuring cough suppression. a) Capsaicin concentration to elicit five coughs during self-attempted suppression of coughing (CS5) in healthy controls; b) CS5 in participants with chronic refractory cough. 
FIGURE 4 The relationships between awake cough count and minimum capsaicin concentrations required to elicit five coughs in tussive challenge tests with self-attempted cough suppression (CS5) in participants with chronic refractory cough.

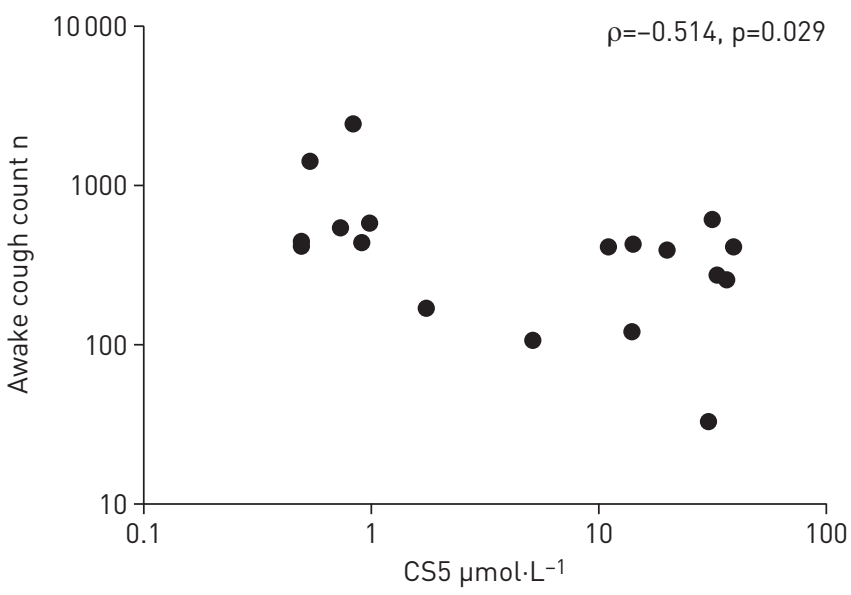

their cough have yielded encouraging results [31, 32]. A recent randomised controlled trial of physiotherapy and speech and language therapy intervention led to a $41 \%$ reduction in cough frequency and an improvement in health-related quality of life [32]. Morphine is an effective antitussive in a subgroup of patients with CRC [33]. It is likely that morphine acts centrally, but it is not known whether it mediates its action by acting on cough amplification or inhibitory pathways. Morphine is associated with side-effects such as drowsiness which limits its use. A better understanding of the central mechanisms of cough may yield new therapeutic targets with a better side-effect profile.

The healthy subjects in our study were able to suppress their cough during a tussive challenge test and this was consistent with the findings of Hutchings et al. [15]. However, a study by BiCKerman et al. [34] in 1956 reported that the cough response following an initial challenge diminishes when the challenge is repeated. This was not tachyphylaxis, since the tests were performed on separate days. This finding has not been confirmed in subsequent, larger studies by WRIGHT et al. [35] and DicPinigaiTis et al. [21], both reporting no significant reduction in cough during sequential challenges. A potential explanation for the contrasting findings is differences in the equipment used and methodology between the studies. WRIGHT et al. [35] compared tussive challenges using a dosimeter with and without an inspiratory flow regulator valve, and found that a diminished cough response with sequential challenges was associated only with the dosimeter with unregulated inspiratory flow. It is possible that subjects in the BICKERMAN et al. [34] study became aware of the stimulus after the initial challenge and that the challenge can become unpleasant at high concentrations of tussive agent. Thus the subject, through a learned response, may not have inhaled as strongly on subsequent inhalations as they did first [35]. This is not possible with the KoKo digidoser we used in our study, because inspiratory flow is regulated and limited $[8,21]$. The flow-volume signals
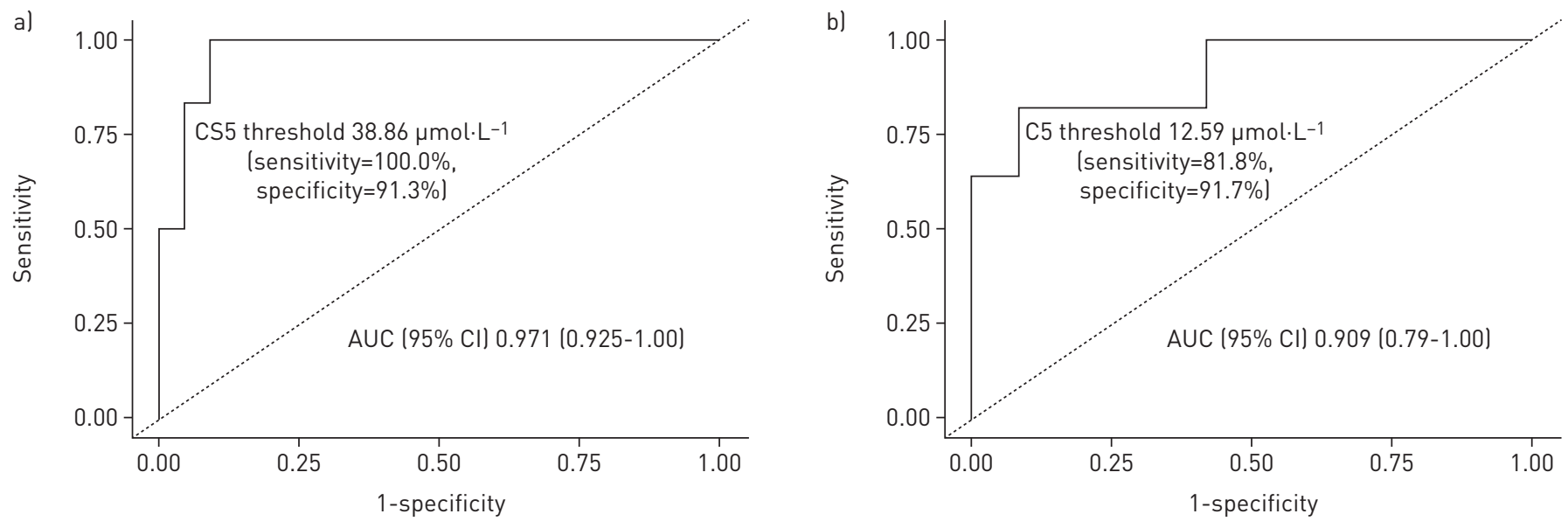

FIGURE 5 Receiver operating characteristic (ROC) curves for capsaicin cough thresholds that distinguish participants with chronic refractory cough from healthy controls. a) ROC curve for cough suppression test end-point (capsaicin concentration to elicit five coughs during self-attempted suppression of coughing (CS5)); b) ROC curve for standard capsaicin challenge end-point (capsaicin concentration to elicit five coughs during capsaicin challenge test without self-attempted suppression (C5)). AUC: area under the ROC curve. 
were inspected in real time by two of the investigators for each inhalation during all tussive challenges. Furthermore, we found CS5 to be highly repeatable, and there was no effect of the order in which challenges were performed. This is further supported by the lack of a test order effect on the cough suppression thresholds in healthy individuals in the study by Hutchings et al. [15]. In addition, we explored the potential for anxiety and mood to influence the ability of subjects to suppress cough. There was no association between CS5 and mood and anxiety levels assessed with validated tools.

Cough in chronic respiratory disease may be driven by several mechanisms such as cough reflex hypersensitivity, airway hyperresponsiveness and airway inflammation [36]. The identification of the mechanism of cough may be useful to target specific therapy and avoid unnecessary trials of treatment [37]. Cough provocation tests are an objective measure of cough reflex sensitivity, but their use in clinical practice has been limited by several factors, one being that they poor at discriminating patients with cough hypersensitivity syndrome from healthy subjects $[8,38]$. To our knowledge, our study is the first to demonstrate that modifying the standard capsaicin tussive challenge by requesting that subjects attempt to suppress their cough makes it a better discriminator of patients with CRC from healthy subjects. The cough suppression test end-point CS5 threshold of $39 \mu \mathrm{mol} \cdot \mathrm{L}^{-1}$ had a high sensitivity and specificity for discriminating between patients with CRC and healthy subjects. Further studies of the CS5 measure in a range of respiratory disorders, such as chronic obstructive pulmonary disease, asthma and idiopathic pulmonary fibrosis, are needed to investigate the clinical and diagnostic usefulness of cough suppression tests. The potential for the cough suppression test to identify patients who respond to centrally acting therapies, such as opiates, should also be investigated.

There are limitations with our study. We studied a relatively small sample size. The order of the capsaicin challenge test with and without voluntary suppression was not randomised. However, we did enrol consecutive patients and, furthermore, there was no significant order effect of the voluntary cough suppression test in the repeatability study. We studied CS5 at the first visit to avoid the potential influence of prior tussive challenges. We found no significant association between CS5 and a subjective measure of health-related quality of life. This may be partly due to the small sample size of our study, but this observation is consistent with numerous studies that have reported a poor correlation between subjective and objective measures of cough $[38,39]$. Cough reflex sensitivity and health-related quality of life are very different dimensions of cough, and as such they are expected to correlate poorly. The sensitivity for C5 to discriminate patients with CRC from healthy controls was lower in our study compared to that reported by Pullerits et al. [40]. This may be due to differences in the study populations and sample sizes; Pullerits et al. [40] recruited participants who had other airway symptoms in addition to cough and they were pre-selected for hyperreactivity to capsaicin.

In conclusion, patients with CRC are unable to suppress their cough during a capsaicin cough challenge test compared to healthy controls. CS5 is a highly repeatable measure and is associated with objective 24-h cough frequency. CS5 has a high sensitivity and specificity for distinguishing patients with CRC from healthy controls. Further studies should investigate the neural pathways involved in inhibiting cough, as this may identify new targets for the development of antitussive therapy.

Acknowledgements: We thank all the patients and volunteers for their participation in the study. We would like to thank Tracey Fleming (Chest Unit) and the staff of the specialist cough clinic at King's College Hospital (London, UK) for their assistance in characterising the patients.

Author contributions: Conception and design: S.S. Birring, R.D. Turner, P.S.P. Cho and H.V. Fletcher; participant screening: S.S. Birring, R.D. Turner, P.S.P. Cho and H.V. Fletcher; study recruitment: S.S. Birring, R.D. Turner, P.S.P. Cho and H.V. Fletcher; data analysis: P.S.P. Cho, S.S. Birring, R.D. Turner and H.V. Fletcher; interpretation of data: P.S.P. Cho, S.S. Birring, R.D. Turner and H.V. Fletcher; manuscript drafting: P.S.P. Cho, S.S. Birring, R.D. Turner and H.V. Fletcher; manuscript revison: S.S. Birring, P.S.P. Cho, R.D. Turner, H.V. Fletcher and C.J. Jolley.

Conflict of interest: P.S.P. Cho reports a grant from Merck outside the submitted work. H.V. Fletcher has nothing to disclose. R.D. Turner has nothing to disclose. C.J. Jolley has nothing to disclose. S.S. Birring reports grants and personal fees for advisory board work from Merck, personal fees for advisory board work from Bayer, GSK, Menlo and Sanofi, and conference travel expenses from Boehringer Ingelheim, outside the submitted work.

\section{References}

$1 \quad$ Irwin RS, Madison JM. The diagnosis and treatment of cough. N Engl J Med 2000; 343: 1715-1721.

2 Song W-J, Chang Y-S, Faruqi S, et al. The global epidemiology of chronic cough in adults: a systematic review and meta-analysis. Eur Respir J 2015; 45: 1479-1481.

3 Haque RA, Usmani OS, Barnes PJ. Chronic idiopathic cough: a discrete clinical entity? Chest 2005; 127: $1710-1713$.

4 Gibson PG, Vertigan AE. Management of chronic refractory cough. BMJ 2015; 351: h5590.

5 French CL, Irwin RS, Curley FJ, et al. Impact of chronic cough on quality of life. Arch Intern Med 1998; 158: $1657-1661$. 
6 McGarvey LPA, Carton C, Gamble LA, et al. Prevalence of psychomorbidity among patients with chronic cough Cough 2006; 2: 4.

7 Dicpinigaitis PV, Tso R, Banauch G. Prevalence of depressive symptoms among patients with chronic cough Chest 2006; 130: 1839-1843.

8 Prudon B, Birring SS, Vara DD, et al. Cough and glottic-stop reflex sensitivity in health and disease. Chest 2005; 127: $550-557$.

9 Mazzone SB, Chung KF, McGarvey L. The heterogeneity of chronic cough: a case for endotypes of cough hypersensitivity. Lancet Respir Med 2018; 6: 636-646.

10 Eccles R. Central mechanisms IV: conscious control of cough and the placebo effect. In: Chung KF, Widdicombe J, eds. Pharmacology and Therapeutics of Cough. Handbook of Experimental Pharmacology. Vol. 187. Berlin, Springer, 2009; pp. 241-262.

11 Hegland KW, Bolser DC, Davenport PW. Volitional control of reflex cough. J Appl Physiol 2012; 113: 39-46.

12 Ando A, Smallwood D, McMahon M, et al. Neural correlates of cough hypersensitivity in humans: evidence for central sensitisation and dysfunctional inhibitory control. Thorax 2016; 71: 323-329.

13 Mazzone SB, Cole LJ, Ando A, et al. Investigation of the neural control of cough and cough suppression in humans using functional brain imaging. J Neurosci 2011; 31: 2948-2958.

14 Leech J, Mazzone SB, Farrell MJ. Brain activity associated with placebo suppression of the urge-to-cough in humans. Am J Respir Crit Care Med 2013; 188: 1069-1075.

15 Hutchings HA, Morris S, Eccles R, et al. Voluntary suppression of cough induced by inhalation of capsaicin in healthy volunteers. Respir Med 1993; 87: 379-382.

16 Hutchings HA, Eccles R, Smith AP, et al. Voluntary cough suppression as an indication of symptom severity in upper respiratory tract infections. Eur Respir J 1993; 6: 1449-1454.

17 Morice AH, McGarvey L, Pavord I. Recommendations for the management of cough in adults. Thorax 2006; 61: Suppl. 1, i1-i24.

18 Morice AH, Higgins KS, Yeo WW. Adaptation of cough reflex with different types of stimulation. Eur Respir J 1992; 5: 841-847.

19 Morice AH, Fontana GA, Belvisi MG, et al. ERS guidelines on the assessment of cough. Eur Respir J 2007; 29: $1256-1276$.

20 O'Connell F, Thomas VE, Studham JM, et al. Capsaicin cough sensitivity increases during upper respiratory infection. Respir Med 1996; 90: 279-286.

21 Dicpinigaitis PV. Short- and long-term reproducibility of capsaicin cough challenge testing. Pulm Pharmacol Ther 2003; 16: 61-65.

22 Birring SS, Fleming T, Matos S, et al. The Leicester Cough Monitor: preliminary validation of an automated cough detection system in chronic cough. Eur Respir J 2008; 31: 1013-1018.

23 Birring SS, Prudon B, Carr AJ, et al. Development of a symptom specific health status measure for patients with chronic cough: Leicester Cough Questionnaire (LCQ). Thorax 2003; 58: 339-343.

24 Kroenke K, Spitzer RL, Williams JBW. The PHQ-9: validity of a brief depression severity measure. J Gen Intern Med 2001; 16: 606-613.

25 Spitzer RL, Kroenke K, Williams JBW, et al. A brief measure for assessing generalized anxiety disorder: the GAD-7. Arch Intern Med 2006; 166: 1092-1097.

26 Miller MR, Hankinson J, Brusasco V, et al. Standardisation of spirometry. Eur Respir J 2005; 26: 319-338.

27 Youden WJ. Index for rating diagnostic tests. Cancer 1950; 3: 32-35.

28 Young EC, Brammer C, Owen E, et al. The effect of mindfulness meditation on cough reflex sensitivity. Thorax 2009; 64: 993-998

29 Abdulqawi R, Dockry R, Holt K, et al. P2X3 receptor antagonist (AF-219) in refractory chronic cough: a randomised, double-blind, placebo-controlled phase 2 study. Lancet 2015; 385: 1198-1205.

30 Canning BJ, Mazzone SB, Meeker SN, et al. Identification of the tracheal and laryngeal afferent neurones mediating cough in anaesthetized guinea-pigs. J Physiol 2004; 557: 543-558.

31 Vertigan AE, Theodoros DG, Gibson PG, et al. Efficacy of speech pathology management for chronic cough: a randomised placebo controlled trial of treatment efficacy. Thorax 2006; 61: 1065-1069.

32 Chamberlain Mitchell SAF, Garrod R, Clark L, et al. Physiotherapy, and speech and language therapy intervention for patients with refractory chronic cough: a multicentre randomised control trial. Thorax 2017; 72: 129-136.

33 Morice AH, Menon MS, Mulrennan SA, et al. Opiate therapy in chronic cough. Am J Respir Crit Care Med 2007; 175: 312-315.

34 Bickerman HA, Cohen BM, German E, et al. The cough response of normal human subjects stimulated experimentally by citric acid aerosol: alterations produced by antitussive agents. I. Methodology. Am J Med Sci 1956; 232: 57-65.

35 Wright CE, Jackson J, Thompson RL, et al. Validation of the ERS standard citric acid cough challenge in healthy adult volunteers. Cough 2010; 6: 8.

36 Birring SS. Controversies in the evaluation and management of chronic cough. Am J Respir Crit Care Med 2011; 183: 708-715.

37 Song WJ, Kim HJ, Shim JS, et al. Diagnostic accuracy of fractional exhaled nitric oxide measurement in predicting cough-variant asthma and eosinophilic bronchitis in adults with chronic cough: a systematic review and meta-analysis. J Allergy Clin Immunol 2017; 140: 701-709.

38 Spinou A, Birring SS. An update on measurement and monitoring of cough: what are the important study endpoints? J Thorac Dis 2014; 6: Suppl. 7, S728-S734.

39 Birring SS, Matos S, Patel RB, et al. Cough frequency, cough sensitivity and health status in patients with chronic cough. Respir Med 2006; 100: 1105-1109.

40 Pullerits T, Ternesten-Hasséus E, Johansson EL, et al. Capsaicin cough threshold test in diagnostics. Respir Med 2014; 108: 1371-1376. 